\title{
Nucleoside Transport Inhibition by Dipyridamole Prevents Angiogenesis Impairment by Homocysteine and Adenosine
}

\author{
Antony Kam ${ }^{\text {a,b,c }}$, Valentina Razmovski-Naumovski ${ }^{\text {a,b,d }}$, Xian Zhou ${ }^{\text {b,c }}$, John Truong ${ }^{\text {b,c }}$, Kelvin Chan ${ }^{\text {a,be }}$ \\ ${ }^{a}$ Faculty of Pharmacy, the University of Sydney, NSW, Australia; ${ }^{b}$ National Institute of Complementary Medicine (NICM), \\ Western Sydney University, NSW, Australia; ${ }^{\mathrm{c}}$ School of Biological Sciences, Nanyang Technological University, \\ Singapore, ${ }^{\mathrm{d}}$ South West Sydney Clinical School, School of Medicine, University of New South Wales, NSW, Australia; \\ ${ }^{\mathrm{e}}$ School of Pharmacy \& Biomolecular Sciences, Liverpool John Moores University, UK..
}

Received, August 8, 2015; Revised, November 26, 2015; Accepted, November 27, 2015; Published, December 8, 2015

\begin{abstract}
Purpose. Adenosine plays an important role in the pathogenesis of homocysteine-associated vascular complications. Methods: This study examined the effects of dipyridamole, an inhibitor for nucleoside transport, on impaired angiogenic processes caused by homocysteine and adenosine in human cardiovascular endothelial cell line (EAhy926). Results: The results showed that dipyridamole restored the extracellular adenosine and intracellular S-adenosylhomocysteine concentrations disrupted by the combination of homocysteine and adenosine. Dipyridamole also ameliorated the impaired proliferation, migration and formation of capillary-like tubes of EAhy926 cells caused by the combination of homocysteine and adenosine. Mechanism analysis revealed that dipyridamole induced the phosphorylation of mitogen-activated protein kinase kinase (MEK) and extracellular signal-regulated kinases (ERK) and its effect on cell growth was attenuated by the MEK inhibitor, U0126. Conclusion: Dipyridamole protected against impaired angiogenesis caused by homocysteine and adenosine, at least in part, by activating the MEK/ERK signalling pathway, and this could be associated with its effects in suppressing intracellular S-adenosylhomocysteine accumulation.

Novelty of the Work: This is the first paper showing that nucleoside transport inhibition by dipyridamole reduced impaired angiogenic process caused by homocysteine and adenosine.
\end{abstract}

This article is open to POST-PUBLICATION REVIEW. Registered readers (see "For Readers") may comment by clicking on ABSTRACT on the issue's contents page.

\section{INTRODUCTION}

Blood vessels form a tubular network to facilitate blood transportations in the circulatory system, and assist distant exchanges of oxygen, carbon dioxide, wastes, electrolytes and hormones from one part of the body to the other. Angiogenesis, defined as the formation of new vessels from existing vasculature, is an essential physiological process for maintaining tissue homeostasis by facilitating nutrient transportation, as well as promoting tissue growth and repair (1). Insufficient angiogenesis can complicate conditions such as myocardial infarction and ischaemic stroke, which are associated with inadequate blood supply (1). Therefore, promoting angiogenesis may be a therapeutic approach for improving the outcome of ischaemic cardiovascular and cerebrovascular diseases.

Homocysteine is a sulfur-containing amino acid derived from methionine metabolism. An accumulation of plasma homocysteine, often referred to as hyperhomocysteinaemia, has been suggested as an independent predictor for ischaemic heart diseases and stroke (2). Hence, hyperhomocysteinaemia is an emerging risk factor for cardiovascular diseases as indicated by the World Health Organization (3). Several reports have highlighted the importance of adenosine in the pathogenesis of vascular complications associated with hyperhomocysteinaemia, where its extracellular level is increased in response to cell injury and stress, such as in ischaemia (4-6). Animal and cellular studies have shown that the combination of homocysteine and adenosine

\footnotetext{
Correspondence Author: Professor Kelvin Chan, NICM, Western Sydney University, NSW, Australia and School of Pharmacy \& Biomolecular Sciences, Liverpool John Moores University, Merseyside, L3 3AF, UK. Tel.: +853 9556 3695. Email: k.k.chan@ljmu.ac.uk
} 
sensitised tumour necrosis factor (TNF) cytotoxicity and apoptosis in endothelial cells, as well as impaired angiogenesis (7-9). In particular, the anti-angiogenic effects of homocysteine and adenosine can be associated with inhibiting extracellular signal-regulated kinases (ERK) and protein kinase B (Akt) signalling pathway (9). Both homocysteine and adenosine are key intermediates involved in the methionine cycle which regulates cellular methylation (10). In the methionine cycle, methylation of DNA, RNA, proteins, phospholipids and other small molecules occurs when Sadenosylmethionine is demethylated to $\mathrm{S}$ adenosylhomocysteine by methyltransferases. Sadenosylhomocysteine is subsequently hydrolysed to homocysteine and adenosine by $\mathrm{S}$ adenosylhomocysteine hydrolase (10). However, when the basal levels of homocysteine and adenosine are high, this favours the accumulation of S-adenosylhomocysteine by reversing the hydrolase reactions of S-adenosylhomocysteine hydrolase. Sadenosylhomocysteine is known to inhibit methyltransferase activities, therefore, the accumulation of S-adenosylhomocysteine can inhibit methyl transfer reactions resulting in cellular hypomethylation (11).

Up till now, there is no recognised treatment regimens for the management of cardiovascular events associated with hyperhomocysteinaemia (12). Understanding the connections between homocysteine and adenosine in the methionine cycle may shed light for novel therapeutic interventions in vascular complications associated with hyperhomocysteinaemia. It is known that a high level of homocysteine induces the synthesis of S-adenosylhomocysteine using the intracellular adenosine pool. This creates a transmembranous adenosine gradient and subsequently, facilitates the cellular uptake of extracellular adenosine $(5,13)$. Dipyridamole is a clinical anti-platelet drug approved for the prevention of thromboembolism, and secondary prevention of ischaemic stroke and transient ischaemic attack. Animal studies have shown that dipyridamole reversed peripheral ischaemia and induced angiogenesis (14). Dipyridamole is known to inhibit the cellular uptake of adenosine by blocking cell surface nucleoside transporters, such as equilibrium nucleoside transporters (ENTs) (15). Considering that adenosine is prominent in vascular complications associated with homocysteine, there is a lack of studies evaluating the potential of nucleoside transport inhibitors in retarding the cellular uptake of adenosine in hyperhomocysteinaemia. Therefore, this study examined the protective effects and mode of actions of a clinically used nucleoside transport inhibitor, dipyridamole, on angiogenesis impairment caused by homocysteine and adenosine in human cardiovascular endothelial cell line (EAhy926) cells.

\section{MATERIALS AND METHODS}

\section{Materials}

U0126, phospho-p44/42 MAPK (ERK1/2) (Thr202/Tyr204) (D13.14.4E), p44/42 MAPK (ERK1/2) (137F5), phospho-MEK1/2 (Ser217/221) (41G9) and MEK1/2 (47E6) antibodies were purchased from Cell Signalling Technology (USA). $\alpha$-Tubulin (DM1A) was purchased from Santa Cruz Biotechnology (USA). CellTiter ${ }^{\circledR} 96$ Aqueous One Solution Cell Proliferation Assay kit was purchased from Promega (Australia). Cultrex Basement Membrane Extract (BME) was purchased from Trevigen (USA). Cell culture reagents were from Life Technology (Australia). Chemicals, including adenosine, dipyridamole, DL-homocysteine, Sadenosylhomocysteine and theophylline were from Sigma-Aldrich (Australia) unless otherwise stated.

\section{Cell line and Culture Conditions}

Human cardiovascular endothelial cell line (EAhy926) was kindly provided by Dr Shanhong Ling (Monash University Central Clinical School, Australia). The cell line was cultured in Dulbecco's modification of Eagle's medium (DMEM)/Ham's F12 containing $15 \mathrm{mM}$ HEPES and L-glutamine and supplemented with $10 \%$ foetal bovine serum, $100 \mathrm{U} / \mathrm{mL}$ of penicillin and streptomycin (Gibco BRL, Australia) (7). The cell line was grown in a $5 \% \mathrm{CO}_{2}$-humidified incubator at $37^{\circ} \mathrm{C}$.

\section{Determination of Extracellular Adenosine Concentration}

To determine the extracellular adenosine concentrations, EAhy926 cells were seeded at a density of $1.0 \times 10^{5}$ cells $/ \mathrm{mL}$ in a 6 well cell culture plate and allowed to adhere and become confluent for $24-48 \mathrm{~h}$. Once confluent, the EAhy926 cells were exposed to homocysteine, adenosine and/or dipyridamole in serum free medium for $3 \mathrm{~h}$. The 
cell culture supernatant was collected and filtered through a $0.22 \mu \mathrm{m}$ syringe filter. The analyses were performed using a Waters Acquity ultra performance liquid chromatography (UPLC) $\mathrm{H}$ series consisting of a $\mathrm{H}$ class quaternary solvent manager, an Acquity sample manager-FTN, an Acquity column oven and an Acquity Photodiode Array detector, scanning from $190-400 \mathrm{~nm}$, and the detection wavelength was set at $254 \mathrm{~nm}$ (Waters, MA, USA). Separation was carried out using a Waters BEH C18 column $(1.7 \mu \mathrm{m}, 2.1 \mathrm{~mm}$ x $150 \mathrm{~mm}$ ) coupled with a BEH guard column (1.7 $\mu \mathrm{m}, 2.1 \mathrm{~mm} \times 5 \mathrm{~mm}$ ) maintained at $40^{\circ} \mathrm{C}$. A binary gradient elution method at a flow rate of $0.3 \mathrm{~mL}$ $\min ^{-1}$ was employed using (A) $0.3 \%$ glacial acetic acid and (B) acetonitrile starting from $0.5 \% \mathrm{~B}$ to $3 \% \mathrm{~B}$ in 3 mins, then to $20 \% \mathrm{~B}$ in 20 mins, back to initial $0.5 \% \mathrm{~B}$ in 3 mins. The system was then reequilibrated at the initial conditions for 5 mins. The total run time was $15 \mathrm{~min}$. All injection volumes of samples and authentic standards were $1 \mu \mathrm{L}$. The identification of adenosine in the supernatant was based on the authentic standards. Retention time and absorbance spectra were used to confirm the presence of adenosine. Quantitative estimations of adenosine were based on calibration curves generated by UPLC-PDA using the adenosine standard. The linear range of the calibration curve used for quantification was $50-1000 \mu \mathrm{M}$.

\section{Determination of Intracellular S- Adenosylhomocysteine Concentration}

To determine the intracellular Sadenosylhomocysteine concentration, EAhy926 cells were seeded at a density of $1.0 \times 10^{5}$ cells $/ \mathrm{mL}$ in a T75 flask and allowed to adhere and become confluent for 24-48 h. Once confluent, the EAhy926 cells were exposed to homocysteine, adenosine and/or dipyridamole in serum-free medium for $3 \mathrm{~h}$. The cells were harvested and washed once with PBS. The cells were then lysed by $100 \mu \mathrm{L}$ of $10 \%$ perchloric acid for 30 mins on ice using sonication. The supernatant was collected by centrifugation at $20,500 \times \mathrm{g}$ for $10 \mathrm{mins}$ at $4^{\circ} \mathrm{C}$. After filtration by a $0.22 \mu \mathrm{m}$ centrifuge tube filter, the supernatant was collected and injected into a Waters Acquity UPLC system ${ }^{\circledR}$, while the pellet was collected and dissolved in $2 \mathrm{M} \mathrm{NaOH}$ for protein quantification using the Bradford reagent. The separation of supernatant was carried out using a Waters BEH C18 column $(1.7 \mu \mathrm{m}, 2.1 \mathrm{~mm}$ x 150 mm) coupled with a BEH guard column $(1.7 \mu \mathrm{m}$, $2.1 \mathrm{~mm} \times 5 \mathrm{~mm}$ ) maintained at $40^{\circ} \mathrm{C}$. A binary gradient elution method at a flow rate of $0.3 \mathrm{~mL}$ $\min ^{-1}$ was employed using (A) $5 \mathrm{mM}$ pentasulfonic acid in $0.1 \%$ phosphoric acid and (B) acetonitrile, starting from $5 \% \mathrm{~B}$ to $20 \% \mathrm{~B}$ in 7 mins, then to $80 \% \mathrm{~B}$ in 3 mins, back to initial 5\% B in 5 mins. The system was re-equilibrated at the initial conditions for 5 mins. The total run time was 20 min. All injection volumes of samples and authentic standards were $1 \mu \mathrm{L}$, and the detection wavelength was set at $254 \mathrm{~nm}$, scanning from $190-400 \mathrm{~nm}$. The identification of S-adenosylhomocysteine was based on the authentic standards. Retention time and absorbance spectra were used to confirm the presence of S-adenosylhomocysteine in the cell extract. The quantitative estimations of Sadenosylhomocysteine were based on calibration curves generated by UPLC-PDA using Sadenosylhomocysteine standard. The linear range of the calibration curve used for quantification was 1 $250 \mu \mathrm{M}$.

\section{Cell Growth Determination}

Cell growth was determined using crystal violet staining and a commercial MTS kit (CellTiter ${ }^{\circledR} 96$ Aqueous One Solution Cell Proliferation Assay) as per manufacturer instructions. Briefly, $1.0 \times 10^{4}$ EAhy926 cells per well were seeded in a 96 well plate for $2 \mathrm{~h}$ and then treated with DLhomocysteine (DL-Hcy), adenosine (Ado) and/or dipyridamole (final DMSO concentration $0.1 \%$ ) in serum free medium. Crystal violet staining was performed as previously described with slight modifications (16). After the incubation period, the wells were fixed with $3 \%$ buffered paraformaldehyde for 20 minutes. The cells were then stained with $0.2 \%$ crystal violet in $20 \%$ methanol for 5 minutes. The excess crystal violet stain was rinsed 4 - 5 times with distilled water and air-dried. Glacial acetic acid (33\%) in Milli-Q water was added to extract the crystal violet stain. The absorbance was measured at $595 \mathrm{~nm}$. For the MTS assay, MTS solution was added and incubated for 2 $\mathrm{h}$ at $37^{\circ} \mathrm{C}$. The absorbance was measured at 490 $\mathrm{nm}$. Cell growth was expressed as a percentage relative to control.

\section{Scratch Wound Healing Assay}

Scratch wound healing assay was performed as previously described with some modifications (17). 
Briefly, EAhy926 cells were seeded at a density of $1.0 \times 10^{5}$ cells $/ \mathrm{mL}$ and allowed to adhere and become confluent for $24-48 \mathrm{~h}$. Once confluent, the EAhy926 cell monolayer was scratched using a $1000 \mu \mathrm{L}$ blue tip, rinsed gently with phosphatebuffered saline (PBS) and then exposed to homocysteine, adenosine and/or dipyridamole in serum free medium. After $24 \mathrm{~h}$ of incubation, the wound was photographed using an inverted phasecontrast light microscope. The percentages of cellfree area were analysed using TScratch software (18).

\section{Tube Formation Assay}

Tube formation assay was performed as previously described with slight modifications (19). Briefly, 50 $\mu \mathrm{L}$ Cultrex ${ }^{\circledR}$ Basement Membrane Extract (BME) per well was used to coat a 96 well flat bottom plate. EAhy926 cells $\left(7.5 \times 10^{3}\right)$ were seeded in each well and incubated for $2 \mathrm{~h}$ and then treated with homocysteine, adenosine and/or dipyridamole in serum free medium. After $24 \mathrm{~h}$ of incubation, the well was photographed using an inverted phasecontrast light microscope. The total number of junctions (consisting of at least three branches) per field was quantified using a plug-in, Angiogenesis Analyzer, developed by Gilles Carpentier in NIH Image-J software (National Institutes of Health, Bethesda, MD).

\section{Western Blot Analysis}

EAhy926 cells were seeded at a density of $1.0 \times 10^{5}$ cells $/ \mathrm{mL}$ in a $60 \mathrm{~mm}$ cell culture dish and allowed to adhere and become confluent for $24-48 \mathrm{~h}$. Once confluent, the EAhy926 cells were exposed to homocysteine, adenosine and/or dipyridamole in serum free medium for $3 \mathrm{~h}$. The cells were harvested and washed with ice-cold PBS after centrifugation at $500 \mathrm{x}$ g for 5 minutes. The cells were then lysed in the commercial radioimmunoprecipitation assay lysis buffer system (Santa Cruz Biotechnology, USA) on ice with frequent agitation for 30 minutes. The cell homogenates were centrifuged at 12,000 rpm for 30 minutes at $4^{\circ} \mathrm{C}$ and the supernatants were collected. Protein concentrations were determined using a micro $\mathrm{BCA}^{\mathrm{TM}}$ Kit (Pierce Chemical, USA). Total protein $(30 \mu \mathrm{g})$ mixed with loading buffer was denatured at $70^{\circ} \mathrm{C}$ for 15 minutes, electrophoretically resolved on iBlot $^{\circledR} 4-12 \%$ BisTris gel (Life Technologies, Australia) and then transferred onto a $0.22 \mu \mathrm{m}$ polyvinylidine difluoride (PVDF) membrane (Amersham, USA). The membranes were incubated for $1 \mathrm{~h}$ at room temperature or overnight at $4{ }^{\circ} \mathrm{C}$ with rabbit primary antibody for p-ERK1/2 (1:1000), ERK1/2 (1:1000), p-MEK1/2 (1:1000), MEK1/2 (1:1000) or mouse $\alpha$ tubulin (1:5000) diluted with $5 \%$ bovine serum albumin (BSA) in tris-buffered saline with Tween 20 (TBST). The membrane was then incubated for $1 \mathrm{~h}$ at room temperature with horseradish peroxidase-conjugated anti-rabbit or anti-mouse secondary antibody (1:2000) (Santa Cruz Biotechnology, USA) diluted with 5\% BSA in TBST. The immune complexes were detected on a X-ray film (Fujifilm Corporation, Japan) using SuperSignal West Pico chemiluminescent substrate (Pierce Chemical, Rockford, IL). For re-probing, the membrane was stripped with guanidine hydrochloride stripping buffer $(6 \mathrm{M}$ guanidine hydrochloride, $0.2 \%$ Nonidet (NP-40), $0.1 \mathrm{M} \beta$ Mercaptoethanol, $20 \mathrm{mM}$ Tris- $\mathrm{HCl}, \mathrm{pH}$ 7.5) twice for 5 minutes, followed by 5 minutes washing with TBST for four times as previously described (20).

\section{Statistical Analyses}

Statistical comparisons were performed using GraphPad Version 5.02 (USA). The data was analysed by one-way analysis of variance (ANOVA) followed by Newman-Keuls post hoc test or Student's t-test for comparing intracellular Sadenosylhomocysteine levels. Data was expressed as mean \pm S.E.M. $p<0.05$ was considered as statistically significant.

\section{RESULTS}

\section{Influence of Dipyridamole on Extracellular Adenosine and Intracellular S- adenosylhomocysteine Concentrations}

It is known that high concentrations of homocysteine can promote Sadenosylhomocysteine formation using the intracellular adenosine pool and drives the uptake of extracellular adenosine (5). Meanwhile, dipyridamole is known to inhibit nucleoside transporters, such as ENT-1 and ENT-2 (15). Therefore, we evaluated the effects of dipyridamole on the extracellular adenosine and intracellular Sadenosylhomocysteine levels following homocysteine and adenosine treatment. The results demonstrated that homocysteine and adenosine 
reduced the extracellular adenosine level from 500 $\mu \mathrm{M}$ to $178 \pm 5.52 \mu \mathrm{M}$ (Fig $1 \mathrm{~A}$ ) with a concomitant increase in intracellular $\mathrm{S}$-adenosylhomocysteine level to $1.19 \pm 0.94 \mu \mathrm{M} / \mathrm{mg}$ protein (Fig 1B). More importantly, simultaneous treatment with dipyridamole significantly prevented the depleted level of extracellular adenosine $(\mathrm{p}<0.05)$ caused by homocysteine and adenosine, and in parallel, significantly reduced the increased intracellular Sadenosylhomocysteine level to $0.10 \pm 0.06 \mu \mathrm{M} / \mathrm{mg}$ protein $(\mathrm{p}<0.05)$.

A
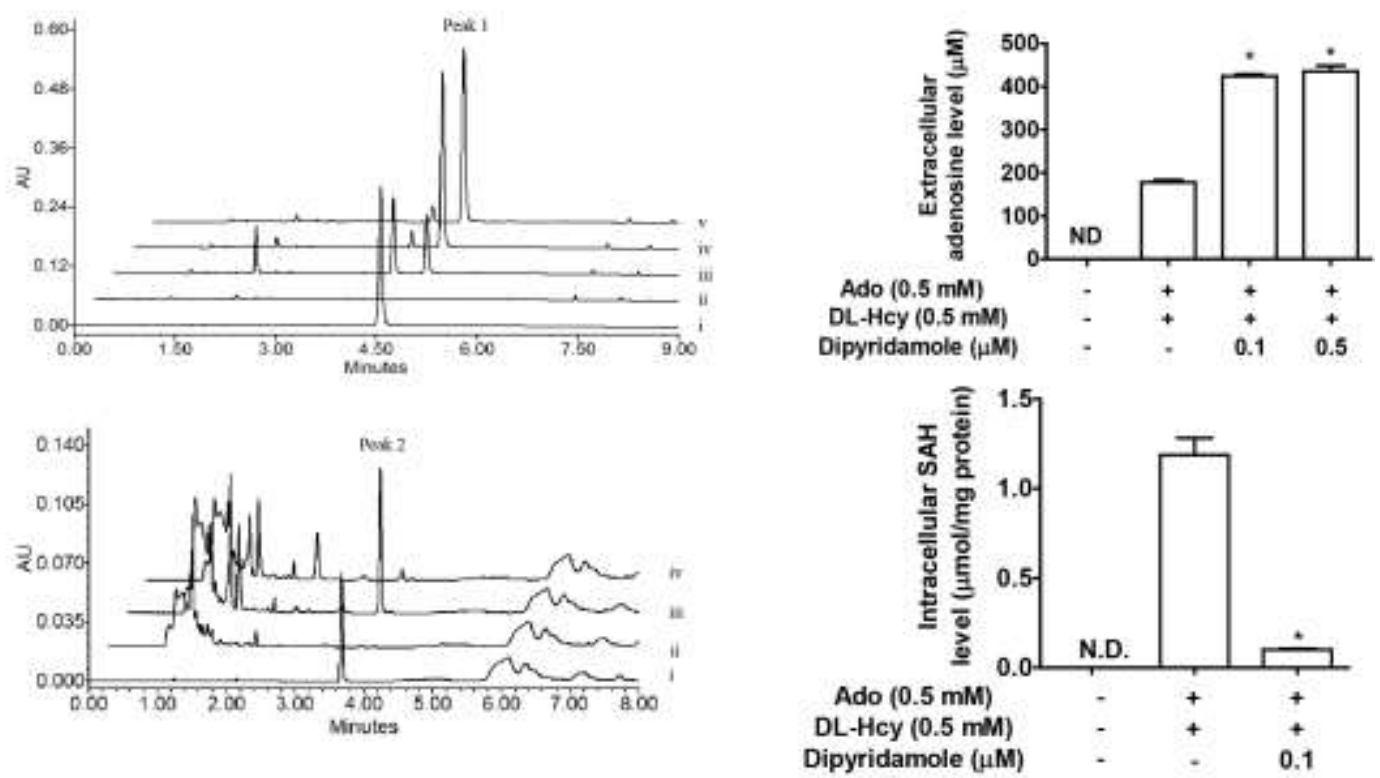

Figure 1. Effects of dipyridamole on extracellular adenosine and intracellular S-adenosylhomocysteine levels in EAhy926 cells treated with DL-Hcy and Ado. EAhy926 cells were exposed to homocysteine (DL-Hcy), adenosine (Ado) and dipyridamole for $3 \mathrm{~h}$ in serum free medium. UPLC-PDA was employed to study the (A) extracellular adenosine level and (B) intracellular S-adenosylhomocysteine (SAH) level in EAhy926 cells following treatment. UPLC-PDA chromatograms of (i) authentic standards of adenosine (peak 1) and S-adenosylhomocysteine (peak 2), (ii) control group, (ii) DL-Hcy plus Ado, (iii) dipyridamole $(0.1 \mu \mathrm{M})$ and (iv) dipyridamole $(0.5 \mu \mathrm{M})$. All results were expressed as mean \pm S.E.M. in triplicate $(n=3)$. ND refers to not detected. ${ }^{*} \mathrm{p}<0.05$ compared to DL-Hcy plus Ado treated-group.

\section{Effects of Homocysteine and Adenosine on Endothelial Cell Growth}

To study the effects of homocysteine and adenosine on angiogenesis, endothelial cell growth was examined. Cell growth measured by crystal violet staining was performed in EAhy926 cells treated with increasing concentrations of homocysteine and adenosine for $96 \mathrm{~h}$. As shown in Fig 2A - B, the results demonstrated that homocysteine promoted a small increment in cell growth at $0.5 \mathrm{mM}$, whereas adenosine (up to $0.5 \mathrm{mM}$ ) showed no significant influences on cell growth. Interestingly, cell growth was significantly attenuated in a concentration- dependent manner when treated with both homocysteine and adenosine $(0.1-0.5 \mathrm{mM})$ (Fig 2C). The results showed that the inhibition in EAhy926 cell growth caused by homocysteine and adenosine was dependent on incubation time and reached a plateau after $72 \mathrm{~h}$ (Fig 2D).

\section{Influence of Dipyridamole on Impaired Endothelial Cell Growth}

To evaluate the effects of dipyridamole on the impaired endothelial cell growth caused by homocysteine and adenosine, EAhy926 cells were treated with increasing concentrations of 
A

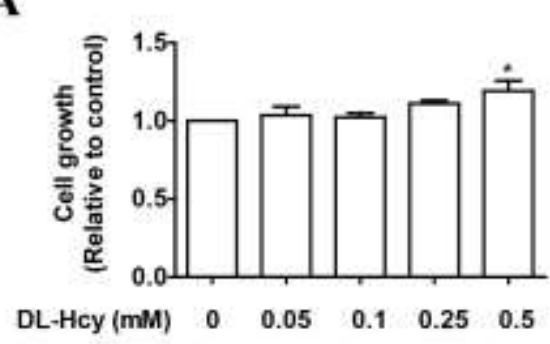

B

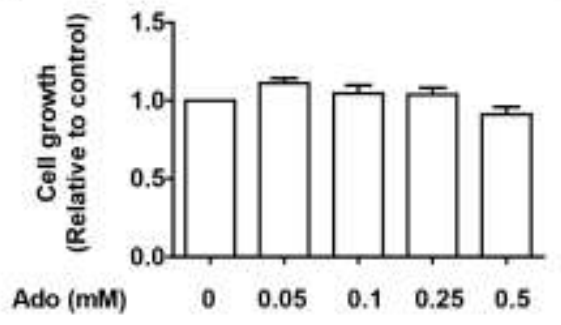

$\mathrm{C}$

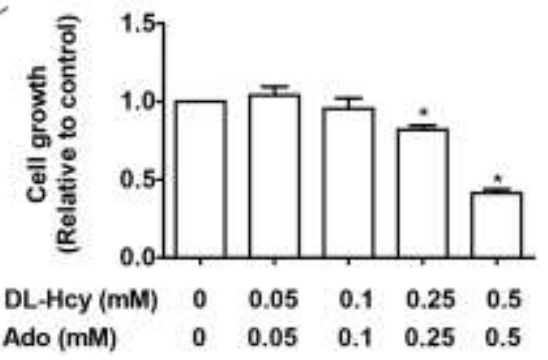

D

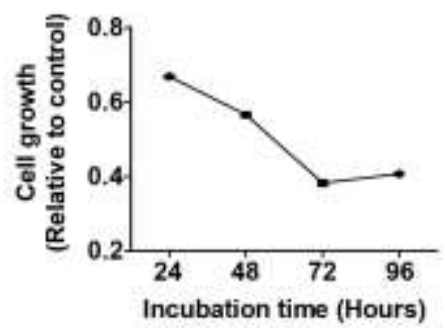

Figure 2. Effects of homocysteine and adenosine on endothelial cell growth. Cell growth, as determined by crystal violet staining, following treatments with (A) homocysteine (DL-Hcy), (B) adenosine (Ado), and (C) DL-Hcy plus Ado in EAhy926 cells for $96 \mathrm{~h}$ in serum free medium. (D) Time-dependent effects of DL-Hcy $(0.5 \mathrm{mM})$ plus Ado $(0.5 \mathrm{mM})$ in serum free medium on the growth of EAhy926 cells. Cell growth was expressed as fold change relative to control. All results were expressed as mean \pm S.E.M. in triplicate $(n=3)$. $* \mathrm{p}<0.05$ compared to control.

A

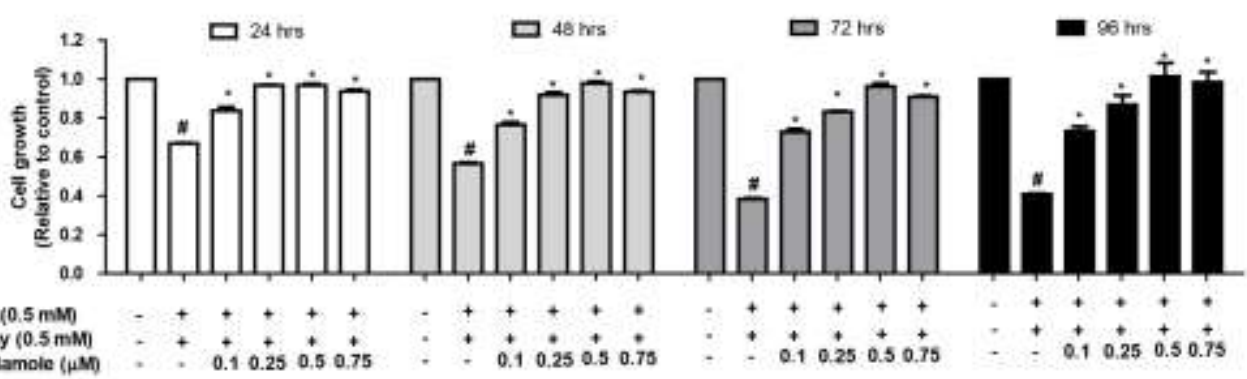

B

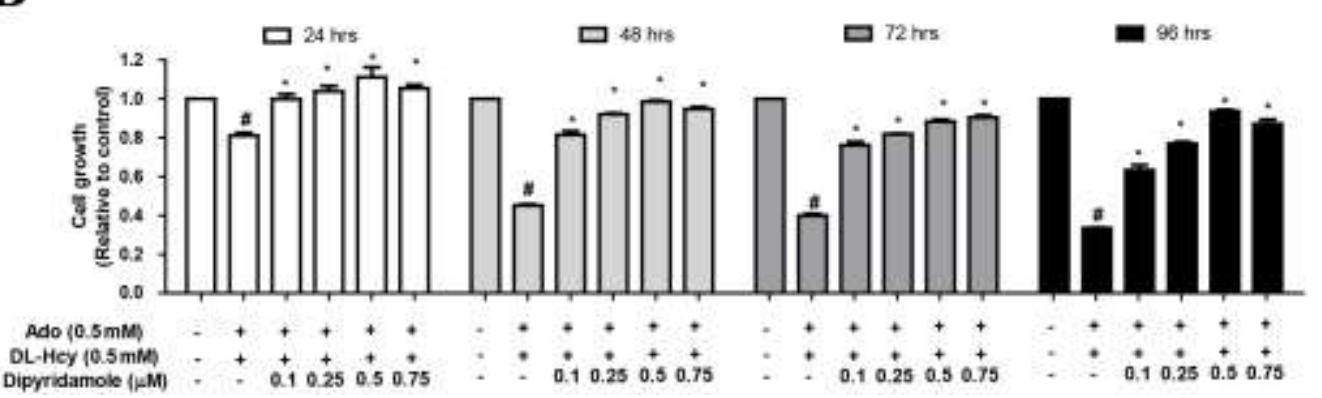

Figure 3. Effects of dipyridamole on impaired endothelial cell growth caused by homocysteine and adenosine. Cell growth, as determined by (A) crystal violet staining and (B) CellTiter ${ }^{\circledR} 96$ Aqueous One Solution Cell Proliferation MTS assay, following treatments with homocysteine (DL-Hcy), adenosine (Ado) and dipyridamole for 24, 48, 72 and $96 \mathrm{~h}$ in serum free medium. All results were expressed as mean \pm S.E.M. in triplicate $(n=3)$. $\# \mathrm{p}<0.05$ compared to control. $* \mathrm{p}<$ 0.05 compared to DL-Hcy plus Ado treated-group. 
dipyridamole $(0.1 \quad-0.75 \mu \mathrm{M})$ together with homocysteine and adenosine for 24, 48, 72 and 96 h. The results from crystal violet staining showed that dipyridamole concentration-dependently increased the growth of EAhy926 cells caused by homocysteine and adenosine (Fig. 3A). Similar results were also observed using MTS assay indicating that dipyridamole improved the growth of EAhy926 cells which were inhibited by homocysteine and adenosine (Fig 3B). To investigate the involvement of adenosine receptor in the protective effects of dipyridamole, EAhy926 cells were incubated with theophylline (nonselective adenosine receptor antagonist), dipyridamole, homocysteine and adenosine for 48 h. It was found that the addition of adenosine receptor antagonist, theophylline, did not inhibit the protective effects of dipyridamole on impaired cell growth caused by homocysteine and adenosine (Fig 4).

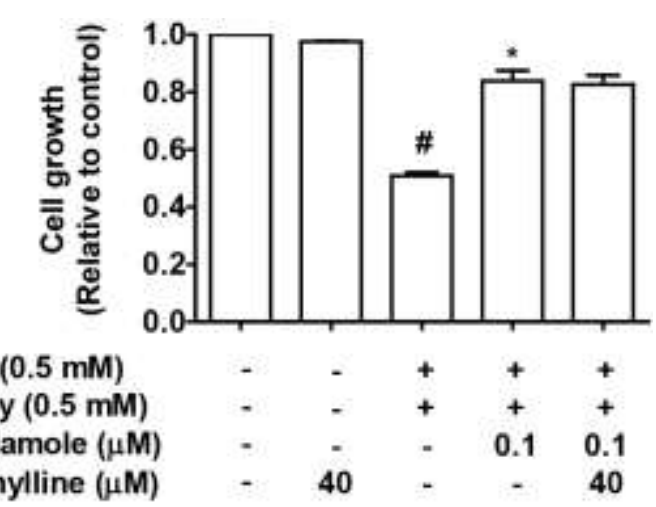

Figure 4. Theophylline did not affect the effects of dipyridamole on impaired endothelial cell growth caused by homocysteine and adenosine. Effects of theophylline (a non-selective adenosine receptor antagonist) on EAhy926 cell growth affected by DL-Hcy and Ado treatment for $48 \mathrm{~h}$ using crystal violet staining. All results were expressed as mean \pm S.E.M. in triplicate $(\mathrm{n}=3) . \# \mathrm{p}<0.05$ compared to control. $* \mathrm{p}<0.05$ compared to DL-Hcy plus Ado treated-group.

\section{Influence of Dipyridamole on Impaired} Endothelial Cell Migration and Tube Formation Scratch wound healing assay was performed to demonstrate the migratory potential of EAhy926 cells following treatment with homocysteine, adenosine and dipyridamole. As shown in Fig 5A, treatment with homocysteine and adenosine significantly attenuated the percentage of scratch wound closure in $24 \mathrm{~h}$, which was subsequently improved with dipyridamole treatment $(p<0.05)$. To model the reendothelisation stage of angiogenesis in vitro, tube formation assay was performed. The results showed that homocysteine and adenosine significantly reduced the total number of junctions per field in EAhy926 cells grown on basement membrane extract compared to the control ( $\mathrm{p}<0.05)$, indicating the inhibition of tube formation (Fig 5B). When the EAhy926 cells were treated simultaneously with dipyridamole, the inhibited tube formation caused by homocysteine and adenosine was significantly restored $(\mathrm{p}<0.05)$.

\section{Influence of Dipyridamole on the Phosphorylation of MEK and ERK}

Previous studies have shown that extracellular adenosine and intracellular Sadenosylhomocysteine levels affect the activation of the MEK/ERK signalling pathway $(21,22)$. The results from immunoblot showed that treatment with homocysteine and adenosine reduced the expressions of p-MEK1/2 and p-ERK1/2 (Fig 6A). Interestingly, when the EAhy926 cells were treated simultaneously with dipyridamole, homocysteine and adenosine, the depleted expressions of $\mathrm{p}$ MEK1/2 and p-ERK1/2 were reversed. U0126 (MEK specific inhibitor) was found to significantly diminish the protective effects of dipyridamole against depleted cell growth induced by homocysteine and adenosine (Fig 6B).

\section{DISCUSSION}

Hyperhomocysteinaemia is an independent factor for the risk of cardiovascular diseases which can lead to defective angiogenesis (2,23). Many publications have focused on the vasotoxic effects of homocysteine, however the exact pathophysiological mechanisms is still unclear (5). More recently, studies have revealed that $S$ adenosylhomocysteine is a more sensitive indicator for the risk of cardiovascular diseases than homocysteine (24). In fact, Sadenosylhomocysteine is a metabolic product from homocysteine and adenosine generated by the reverse reactions of $S$-adenosylhomocysteine hydrolase. Therefore, adenosine has been linked to the pathogenesis of vascular complications implicating hyperhomocysteinaemia (5), and the 


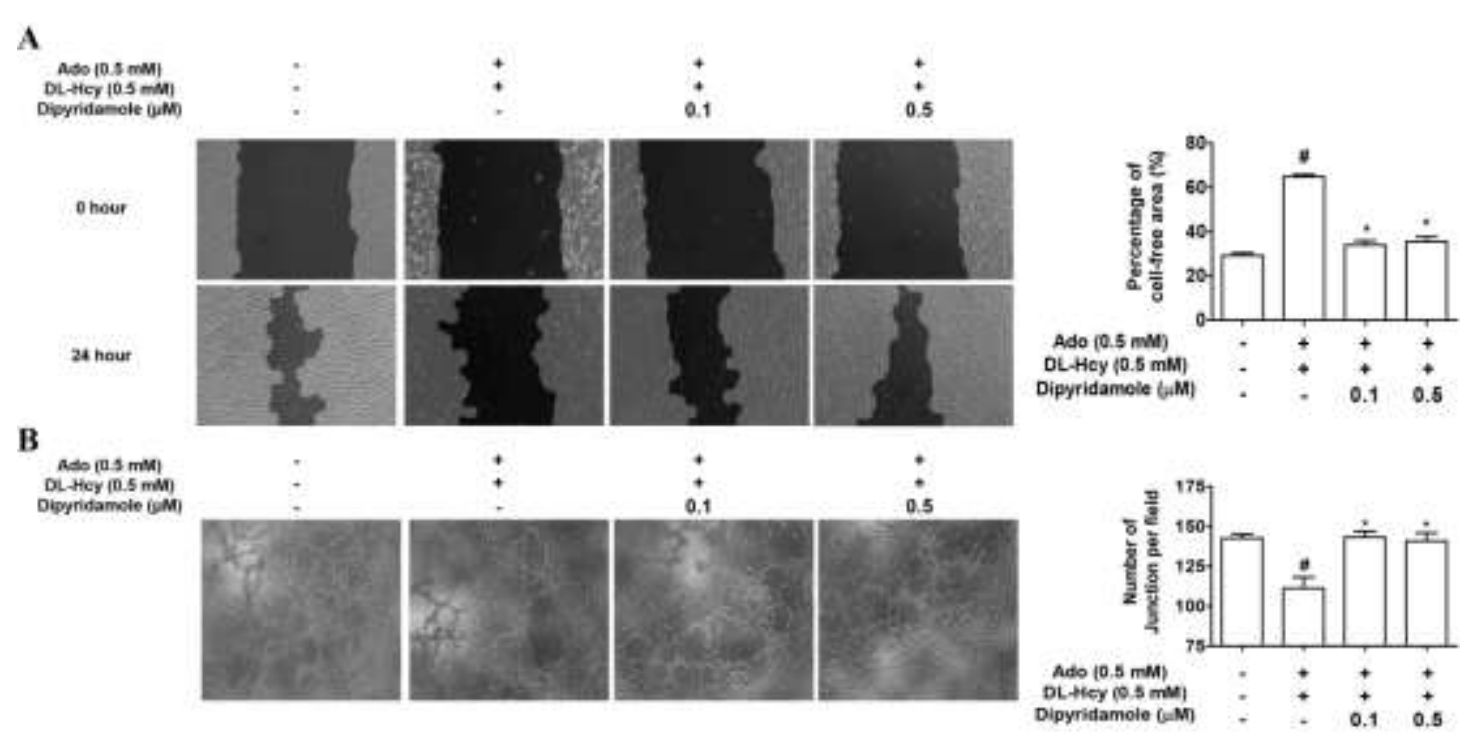

Figure 5. Effects of dipyridamole on impaired wound healing and tube formations in EAhy926 cells induced by homocysteine and adenosine. Wound healing and tube formations were analysed in EAhy926 cells which were exposed to homocysteine (DL-Hcy), adenosine (Ado) and dipyridamole for $24 \mathrm{~h}$ in serum free medium. (A) The results from scratch wound healing assay were presented as the percentage of scratch wound closure in $24 \mathrm{~h}$. (B) The results from tube formation assay were presented as the total number of junctions (consisting of at least three branches) per field. All results were expressed as mean \pm S.E.M. $(n=3)$. \# $p<0.05$ compared to control. $* \mathrm{p}<0.05$ compared to DL-Hcy plus Ado treatedgroup.

A

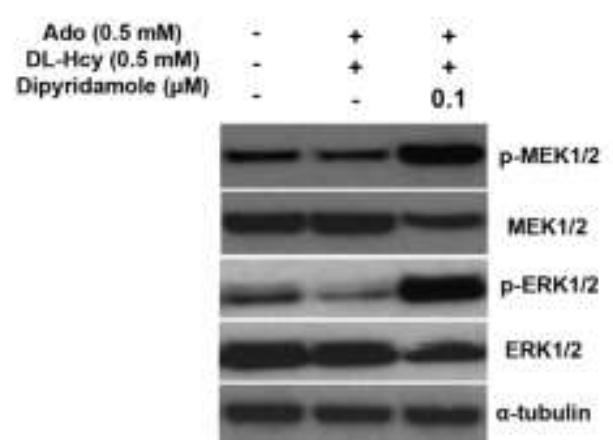

B

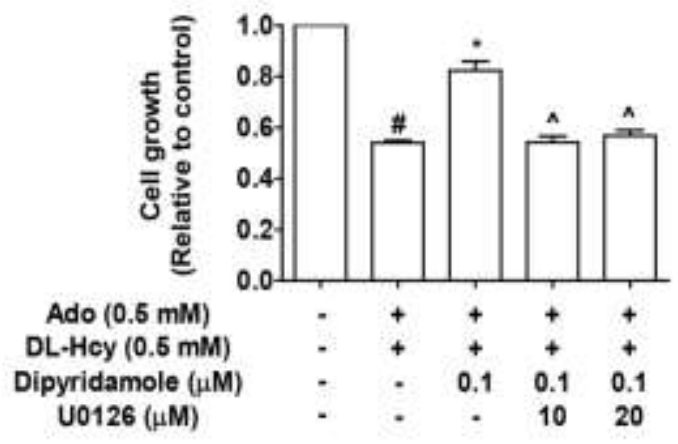

Figure 6. Effects of dipyridamole on the activation of MEK/ERK signalling pathway in EAhy926 cells treated with homocysteine and adenosine. EAhy926 cells which were exposed to homocysteine (DL-Hcy), adenosine (Ado) and dipyridamole for $3 \mathrm{~h}$ in serum free medium. (A) Representative western blot analysis on the phosphorylated MEK1/2 (pMEK1/2), total MEK (MEK1/2), phosphorylated ERK1/2 (p-ERK1/2) and total ERK1/2 (ERK1/2) expressions in EAhy926 cells. (B) Effects of U0126 (a MEK specific inhibitor) on EAhy926 cell growth affected by DL-Hcy and Ado. EAhy926 cells were exposed to DL-Hcy, Ado, dipyridamole and U0126 for $48 \mathrm{~h}$ in serum free medium. Cell growth was analysed by crystal violet staining. All results were expressed as mean \pm S.E.M. in triplicate $(n=3)$. $\# p<0.05$ compared to control. ${ }^{*} \mathrm{p}<0.05$ compared to DL-Hcy plus Ado treated-group. ${ }^{\wedge} \mathrm{p}<0.05$ compared to dipyridamole treated-group.

combination of homocysteine and adenosine has been reported to impair angiogenesis in cellular and zebrafish models (9). Our previous reports have demonstrated that the combination of homocysteine and adenosine exacerbated TNF cytotoxicity and apoptosis in endothelial cells $(7,8)$. This connection between homocysteine and adenosine has been associated with their active involvements in 
methionine metabolism and cellular methylation, as well as the enhanced cellular uptake of adenosine induced by homocysteine (13). To the best of our knowledge, there is a lack of studies evaluating the potential use of nucleoside transport inhibitors to block cellular adenosine uptake in vascular complications associated with homocysteine. In the present study, we show for the first time that a clinically used nucleoside transport inhibitor, dipyridamole, blocked the impairment in angiogenic processes caused by homocysteine and adenosine through the activation of the MEK/ERK signalling pathway.

Accumulating evidence has suggested that therapeutic angiogenesis can re-vascularise ischaemic tissues to improve nutrient perfusion under pathological situations (25). This could potentially alleviate the occurrence and severity of ischaemic symptoms, and ultimately improve functional recovery following ischaemic attacks, including myocardial infarction and stroke $(26,27)$. Angiogenesis is often studied in vitro using vascular endothelial cells focussing on cell growth, migration and capillary-like tube formation (28). Similar to previous findings, our results demonstrated that endothelial cell growth, migration and tube formation were compromised following treatment with homocysteine and adenosine (9). With the addition of dipyridamole, these impaired angiogenic processes, caused by the combination of homocysteine and adenosine, were significantly improved.

The mitogen-activated protein kinase (MAPK) cascade is an important intracellular signal transduction pathway involved in many cellular processes, such as cell growth, differentiation, motility and survival (29). The MAPK cascade is controlled by a sequential protein phosphorylation of three main protein kinases i.e. MAPK kinase kinase (MAPKKK), MAPK kinase (MAPKK) and MAPK (30). ERK1/2, c-Jun amino-terminal kinases (JNK), p38 kinases and ERK5 are among the four well-characterised mammalian MAPK (30). In particular, the activation of Raf/MEK/ERK pathway has been highlighted to play an important role in the process of angiogenesis (31). In the ERK pathway, Raf kinases are the MAPKKK component that phosphorylate the MAPKK components MEK1/2 (30). This, in turn, phosphorylates and activates the MAPK component ERK1/2 which are responsible for stimulating many downstream effector proteins
(30). In this study, our results showed that the combined incubation of homocysteine and adenosine inhibited MEK/ERK signalling pathway which agrees with previous reports (9). Based on this observation, we studied the effects of dipyridamole on the MEK/ERK pathway by using immunoblot and a specific MEK inhibitor (U0126). We demonstrated that dipyridamole activated the homocysteine and adenosine-impaired MEK/ERK signalling pathway. Using the specific MEK inhibitor, we showed that the proliferative effects of dipyridamole in the EAhy926 cells were hindered. Thus, the activation of the MEK/ERK signalling pathway is, at least in part, involved in the angiogeneic effect of dipyridamole.

Previous studies have showed that homocysteine attenuated ischaemia-induced extracellular adenosine accumulation, and this could interfere with the vasoactive response of adenosine (6). Dipyridamole is known as an inhibitor for the cellular uptake of adenosine, and has been shown to preserve the vasodilative effects of adenosine in patients with hyperhomocysteinaemia $(13,15)$. Therefore, the effects of dipyridamole in enhancing angiogenesis and activating MEK/ERK signalling pathway could also be related to its abilities in regulating extracellular adenosine and intracellular Sadenosylhomocysteine concentrations. These biochemical alterations may provide an explanation for the impaired angiogenesis induced by homocysteine and adenosine. The accumulation of intracellular S-adenosylhomocysteine has been previously reported to diminish the proliferative ability of endothelial cells (32), whereas extracellular adenosine can induce adenosine receptor activation, stimulate vascular endothelial growth factor expressions and promote angiogenesis $(33,34)$. In terms of MEK/ERK signalling pathway, the effects of extracellular adenosine and intracellular Sadenosylhomocysteine concentrations on its activations have been previously reported. Intracellular S-adenosylhomocysteine accumulation inhibited the activities of isoprenylcysteine carboxyl methyltransferase and repressed the carboxyl methylation of Ras and its membrane translocation, leading to the inhibition of the cRaf/MEK/ERK signalling pathway (21). Meanwhile, the increase in extracellular adenosine concentration could lead to adenosine receptor 
activation. Previous reports have demonstrated that adenosine receptors are coupled to MAPK signalling pathways, thereby the activation of the adenosine receptor can induce the phosphorylation and activation of MEK and ERK (22). In the present study, dipyridamole increased extracellular adenosine and lowered intracellular Sadenosylhomocysteine levels in EAhy926 cells. However, the protective effects of dipyridamole on impaired endothelial cell growth were not counteracted by theophylline, which is a nonselective adenosine receptor antagonist. The results suggested that the angiogeneic effects of dipyridamole may be less associated with adenosine receptor activation as a result of extracellular adenosine accumulation. For these reasons, we speculated that the angiogeneic effects and activation of the MEK/ERK signalling pathway could be explained by the effects of dipyridamole in suppressing intracellular S-adenosylhomocysteine accumulation.

In summary, the nucleoside transport inhibitor, dipyridamole prevented impairment of angiogenic processes caused by homocysteine and adenosine, at least in part, by activating the MEK/ERK signalling pathway. This can be associated with the effects of dipyridamole in suppressing intracellular S-adenosylhomocysteine accumulation. These findings support dipyridamole as a potential therapeutic candidate for homocysteine-associated vascular complications in addition to its clinically proven anti-platelet property.

\section{CONFLICT OF INTEREST}

The authors declare that they have no conflicts of interests.

\section{ACKNOWLEDGMENTS}

The authors are grateful to Prof. Alan Bensoussan and the facilities in the Herbal Analysis and Pharmacology Laboratories at NICM, Western Sydney University Australia. Prof. Kelvin Chan and Dr Valentina Razmovski-Naumovski were financially supported by The Joint Chair in Traditional Chinese Medicine Program (JCTCM), funded by the Office of Science Research, the University of Sydney and Western Sydney University, New South Wales, Australia. This work was partly supported by the Western Sydney University Research Grant Scheme (P00021202).

\section{REFERENCES}

1. Potente M, Gerhardt H, Carmeliet P. Basic and therapeutic aspects of angiogenesis. Cell 2011; 146: 873-887

2. Homocysteine Studies Collaboration. Homocysteine and risk of ischemic heart disease and stroke: a meta-analysis. JAMA 2002; 288: 2015-2022

3. Mendis S, Puska P, Norrving B. Global Atlas on Cardiovascular Disease Prevention and Control. In. Geneva: World Health Organisation; 2011

4. Li RW, Yang C, Sit AS, Lin SY, Ho EY, Leung GP. Physiological and pharmacological roles of vascular nucleoside transporters. J Cardiovasc Pharmacol 2012; 59: 10-15

5. Riksen NP, Rongen GA, Blom HJ, Russel FG, Boers $\mathrm{GH}$, Smits P. Potential role for adenosine in the pathogenesis of the vascular complications of hyperhomocysteinemia. Cardiovasc Res 2003; 59: 271-276

6. Sciotti VM, Van Wylen DG. Attenuation of ischemia-induced extracellular adenosine accumulation by homocysteine. J Cereb Blood Flow Metab 1993; 13: 208-213

7. Kam A, Li KM, Razmovski-Naumovski V, Nammi S, Chan K, Li GQ. Combination of TNF-alpha, homocysteine and adenosine exacerbated cytotoxicity in human cardiovascular and cerebrovascular endothelial cells. Cell Physiol Biochem 2012; 30: 805-814

8. Kam A, Li KM, Razmovski-Naumovski V, Nammi S, Chan K, Li GQ. Gallic acid protects against endothelial injury by restoring the depletion of DNA methyltransferase 1 and inhibiting proteasome activities. Int J Cardiol 2014; 171: 231-242

9. Zhang Q, Li Q, Chen Y, Huang X, Yang IH, Cao L, Wu WK, Tan HM. Homocysteine-impaired angiogenesis is associated with VEGF/VEGFR inhibition. Front Biosci (Elite Ed) 2012; 4: 25252535

10. Ulrey CL, Liu L, Andrews LG, Tollefsbol TO. The impact of metabolism on DNA methylation. Hum Mol Genet 2005; 14 Spec No 1: R139-147

11. James SJ, Melnyk S, Pogribna M, Pogribny IP, Caudill MA. Elevation in S-adenosylhomocysteine and DNA hypomethylation: potential epigenetic mechanism for homocysteine-related pathology. J Nutr 2002; 132: 2361S-2366S

12. Marti-Carvajal AJ, Sola I, Lathyris D, Karakitsiou DE, Simancas-Racines D. Homocysteine-lowering interventions for preventing cardiovascular events. Cochrane Database Syst Rev 2013; 1: CD006612 
13. Riksen NP, Rongen GA, Boers GH, Blom HJ, van den Broek PH, Smits P. Enhanced cellular adenosine uptake limits adenosine receptor stimulation in patients with hyperhomocysteinemia. Arterioscler Thromb Vasc Biol 2005; 25: 109-114

14. Pattillo CB, Bir SC, Branch BG, Greber E, Shen X, Pardue S, Patel RP, Kevil CG. Dipyridamole reverses peripheral ischemia and induces angiogenesis in the $\mathrm{Db} / \mathrm{Db}$ diabetic mouse hind-limb model by decreasing oxidative stress. Free Radic Biol Med 2011; 50: 262-269

15. Wang C, Lin W, Playa H, Sun S, Cameron K, Buolamwini JK. Dipyridamole analogs as pharmacological inhibitors of equilibrative nucleoside transporters. Identification of novel potent and selective inhibitors of the adenosine transporter function of human equilibrative nucleoside transporter 4 (hENT4). Biochem Pharmacol 2013; 86: 1531-1540

16. Martin A, Clynes M. Comparison of 5 microplate colorimetric assays for in vitro cytotoxicity testing and cell proliferation assays. Cytotechnology 1993; 11: $49-58$

17. Liang CC, Park AY, Guan JL. In vitro scratch assay: a convenient and inexpensive method for analysis of cell migration in vitro. Nat Protoc 2007; 2: 329-333

18. Geback T, Schulz MM, Koumoutsakos P, Detmar M. TScratch: a novel and simple software tool for automated analysis of monolayer wound healing assays. Biotechniques 2009; 46: 265-274

19. Arnaoutova I, Kleinman HK. In vitro angiogenesis: endothelial cell tube formation on gelled basement membrane extract. Nat Protoc 2010; 5: 628-635

20. Yeung YG, Stanley ER. A solution for stripping antibodies from polyvinylidene fluoride immunoblots for multiple reprobing. Anal Biochem 2009; 389: 89-91

21. Kim JH, Kim SC, Yi YS, Yang WS, Yang Y, Kim HG, Lee JY, Kim KH, Yoo BC, Hong S, Cho JY. Adenosine dialdehyde suppresses MMP-9-mediated invasion of cancer cells by blocking the Ras/Raf1/ERK/AP-1 signaling pathway. Biochem Pharmacol 2013; 86: 1285-1300

22. Schulte G, Fredholm BB. Signalling from adenosine receptors to mitogen-activated protein kinases. Cell Signal 2003; 15: 813-827
23. Duan J, Murohara T, Ikeda H, Sasaki K, Shintani S, Akita T, Shimada T, Imaizumi T. Hyperhomocysteinemia impairs angiogenesis in response to hindlimb ischemia. Arterioscler Thromb Vasc Biol 2000; 20: 2579-2585

24. Wagner C, Koury MJ. S-Adenosylhomocysteine: a better indicator of vascular disease than homocysteine? Am J Clin Nutr 2007; 86: 1581-1585

25. Freedman SB, Isner JM. Therapeutic angiogenesis for coronary artery disease. Ann Intern Med 2002; 136: 54-71

26. Sun Y, Jin K, Xie L, Childs J, Mao XO, Logvinova A, Greenberg DA. VEGF-induced neuroprotection, neurogenesis, and angiogenesis after focal cerebral ischemia. J Clin Invest 2003; 111: 1843-1851

27. Cochain C, Channon KM, Silvestre JS. Angiogenesis in the infarcted myocardium. Antioxid Redox Signal 2013; 18: 1100-1113

28. Staton CA, Reed MW, Brown NJ. A critical analysis of current in vitro and in vivo angiogenesis assays. Int J Exp Pathol 2009; 90: 195-221

29. Pearson G, Robinson F, Beers Gibson T, Xu BE, Karandikar M, Berman K, Cobb MH. Mitogenactivated protein (MAP) kinase pathways: regulation and physiological functions. Endocr Rev 2001; 22: 153-183

30. Roberts PJ, Der CJ. Targeting the Raf-MEK-ERK mitogen-activated protein kinase cascade for the treatment of cancer. Oncogene 2007; 26: 3291-3310

31. Murphy DA, Makonnen S, Lassoued W, Feldman MD, Carter C, Lee WM. Inhibition of tumor endothelial ERK activation, angiogenesis, and tumor growth by sorafenib (BAY43-9006). Am J Pathol 2006; 169: 1875-1885

32. Yu X, Ling W, Mi M. Relationship of impairment induced by intracellular S-adenosylhomocysteine accumulation with DNA methylation in human umbilical vein endothelial cells treated with 3deazaadenosine. Int J Exp Pathol 2009; 90: 638-648

33. Linden J. Adenosine in tissue protection and tissue regeneration. Mol Pharmacol 2005; 67: 1385-1387

34. Takagi H, King GL, Robinson GS, Ferrara N, Aiello LP. Adenosine mediates hypoxic induction of vascular endothelial growth factor in retinal pericytes and endothelial cells. Invest Ophthalmol Vis Sci 1996; 37: 2165-2176 